Horizons philosophiques

\title{
Les sentiers de la Caverne ou Petite musique pour Platon
}

\section{Marc Chabot}

Volume 9, numéro 2, printemps 1999

La philosophie à portée de voix

URI : https://id.erudit.org/iderudit/801122ar

DOI : https://doi.org/10.7202/801122ar

Aller au sommaire du numéro

\section{Éditeur(s)}

Collège Édouard-Montpetit

\section{ISSN}

1181-9227 (imprimé)

1920-2954 (numérique)

Découvrir la revue

\section{Citer cet article}

Chabot, M. (1999). Les sentiers de la Caverne ou Petite musique pour Platon. Horizons philosophiques, 9(2), 1-19. https://doi.org/10.7202/801122ar d'utilisation que vous pouvez consulter en ligne.

https://apropos.erudit.org/fr/usagers/politique-dutilisation/ 


\section{LES SENTIERS DE LA CAVERNE OU PETITE MUSIQUE POUR PLATON}

Toute musique est un élan du cœur.

Par la musique, il se produit ce qui ne se produit jamais dans le réel. Un enchantement. Le monde est plus léger. Une joie unique. Une rue, une ville peuvent se mettre à danser. Par la musique, le monde change. Une transformation impossible à expliquer.

La philosophie peut-elle être un enchantement? Oui.

Rarement. Mais il faut répondre oui.

Là également il se passe quelque chose qui ne se produit jamais dans le réel. Le monde change. Sa compréhension devient possible.

Musique et philosophie se rejoignent dans l'enchantement. Le corps et l'esprit peuvent danser.

La musique danse au-dessus du monde.

L'illusion est plus grande.

Peut-être.

Musique et philosophie permettent à l'être d'ouvrir les yeux.

La philosophie change le monde. Ce qui change, ce n'est qu'un concept, mais il ne faut jamais sous-estimer l'importance de ce changement. Les concepts sont comme des notes. C'est ce qu'on ne voit pas et qui permet une autre existence que la nôtre. Ce qui nous fait humains : notes et concepts.

L'humanité commence. 
Dans ce texte, je confondrai intentionnellement musique et chanson. S'il faut approfondir les deux concepts, je le ferai ailleurs, plus tard, une autre fois.

Une chanson est une petite histoire. Un roman en miniature. Ce qui prime, c'est l'économie des mots. Dire brièvement une souffrance, un bonheur, le goût d'être, une rencontre. Sur ces mots s'ajoute une mélodie. De la peine ou de la joie qui se collent au réel. Un tout petit instant pour raconter un morceau de la vie. Une vie devient toutes les vies. Jef de Jacques Brel rend l'amitié visible. Jef de Jacques Brel explique l'amitié, la solitude, I'horreur de la fin d'un amour, l'abandon.

Une chanson est le lieu d'où deviennent possibles et pensables nos premières universalisations.

Nous étions quatre dans une pièce, nous écoutions Jacques Brel et nous étions tous Jef.

Les philosophies se réalisaient dans des traités. Nietzsche a jeté à la poubelle tous les traités.

Les mots se sont mis à danser.

Le philosophe pouvait devenir un artiste. II en était un depuis Platon. Mais l'idée était confuse. L'Allégorie de la Caverne est l'œuvre d'un artiste qui ne voulait pas se dire artiste.

Le philosophe n'est pas parvenu à mettre fin à tous les mythes. II avait besoin des légendes pour expliquer. Comme avant les Présocratiques. Platon écrit un traité du monde. Mais son traité est inachevé et inachevable.

Nietzsche signe l'arrêt de mort du traité philosophique comme seul mode d'écriture pour les philosophes.

Le philosophe consent à la poésie. Son écriture devient musicale. 
Si la raison peut édifier le raisonnable,

L'artiste ne doit que digérer l'art,

Et pourtant un artiste a écrit ce livre :

À qui le doit-il? Pas à sa raison, mais à son amour!!

La bataille est engagée. Depuis longtemps. La raison et l'amour s'engueulent. La raison et l'amour : un couple mal assorti. Raison fut mâle, Amour fut femme. Raison fut philosophie. Amour fut poésie. On croyait ainsi les contenir.

L'homme assassine une part de lui-même : sa passion. La femme doit étouffer en elle une part d'elle-même : sa raison. On a voulu, pour simplifier le monde, rendre cette séparation irrémédiable. Génétique, biologique, naturelle. Hermès contre les Muses.

Socrate dans sa caverne.

Sappho sur son île.

En une seule journée nous pouvons entendre des dizaines de chansons. Par elles, sans le savoir, on nous rappelle que nous vivons et que l'amour fait et défait les êtres. Mais nous n'écoutons pas toujours les chansons qui nous sont imposées par la radio. Nous sommes agressés chaque jour.

II n'y a rien entre la philosophie et la musique. Rien qui puisse les séparer. Ce sont des instruments pour dire le plaisir, la peur, la peine, le rire. Ce qui vient en nous et ce qui veut en sortir.

J'ai écrit ma première chanson avec Richard Séguin il y a exactement dix ans. C'était un hommage à l'écrivain Jack Kérouac. L'ange vagabond 2.

1. Friedrich Nietzsche, Poésies, traduites de l'allemand par Georges RibemontDessaignes, Paris : Éditions Gérard Lebovici, 1984, p. 39. Ce livre dont parle le poème, c'est Humain, trop humain.

2. Paroles de Marc Chabot, musique de Richard Séguin et Marie-Claire Séguin.

Sur l'album de Richard Séguin intitulé Journée d'Amérique, Disques

Audiogram, 1988. 
II faut traduire en langage ce que nous sommes. La chanson est aussi une traduction de ce que nous sommes. Tout est traduction. Passage d'un langage à un autre. Est-ce que la langue philosophique peut se traduire en une autre langue?

Je travaillais déjà depuis plusieurs mois avec la chanteuse Claire Pelletier. Nous préparions un disque. Claire est une amoureuse de l'histoire. De toutes les histoires. Celles de sa région, celles de son pays, celles du monde entier.

Elle m'avait dit :

- Je te rejoins à la maison pour souper.

Dans l'après-midi, j'avais un cours de philosophie.

Pendant le repas, elle m'a demandé :

- De quoi parlais-tu durant ton cours aujourd'hui?

- De Platon, plus précisément de L'Allégorie de la Caverne.

- C'est quoi?

- Des hommes sont enchaînés dans une caverne. Ils ne peuvent pas bouger. Ils regardent le mur au fond de la caverne. Ils voient des ombres qui bougent. Ils n'ont jamais rien vu d'autre. Ils ne savent pas qu'ils n'ont accès qu'à l'ombre des choses. Ils ne savent pas qu'il y a une lumière derrière. Ils pensent que les ombres sont le réel. Pour eux, l'ombre est un fait. On ne questionne pas les faits.

Un homme brise ses chaînes. Péniblement il se retourne. II monte vers la lumière. II est aveuglé mais il poursuit sa remontée. II découvre que le réel est ailleurs. II revient vers les autres pour les convaincre de l'imiter. Mais la vie au fond de la caverne est confortable. Les autres résistent. lls ne veulent pas changer leurs habitudes.

Connaître, c'est jeter une lumière sur quelque chose. Modification de notre regard. Modification de l'éclairage. La connaissance et la lumière vont ensemble.

- C'est une très belle histoire, tu devrais m'en faire une chanson. 
Claire ferait une chanson de tout l'univers si elle le pouvait.

Tout en ce monde se transforme en larmes ou en chants.

Faire entendre la voix de Platon en dehors des livres. II ne s'agissait pas de vulgariser Platon, mais de faire entendre un texte en utilisant la chanson comme véhicule. La chanson devenant un moyen autre pour répandre encore plus de lumière.

L'Allégorie de la Caverne est sortie depuis longtemps de La République. Dans les cégeps, les élèves l'ont des centaines de fois dessinée. C'est un peu comme si on leur demandait de dessiner le savoir. Le premier moment de la connaissance. L'ouverture des yeux. L'ouverture au monde.

Un espoir derrière ce travail : celui d'amener quelqu'un à Platon, aux livres, à la connaissance. II y a toujours des chaînes autour de nos cous. Apprendre à se retourner. Apprendre à vivre avec la souffrance du savoir.

La Caverne est aujourd'hui la télévision. Elle enchaîne tous les regards. La Caverne est aujourd'hui une télévision. Sous prétexte de nous montrer le réel, elle finit par nous endormir dans son réel. Elle nous vole nos yeux et du même coup notre humanité.

Elle est, dit-on, une fenêtre sur le monde. Peut-être. Mais elle ne nous dit pas qu'il y a d'autres fenêtres. Celle qui donne sur la rue. Celle qui donne sur le silence d'un matin d'hiver. Celle qui donne sur la conscience de vivre.

Même nos oreilles donnent à voir.

Une chanson est là pour ça.

La philosophie ne se vulgarise pas. Elle est une fenêtre aux mille paysages.

Le mythe de la Caverne est un texte fondateur. Voilà l'humanité dans sa nudité. Voilà l'humanité dans toute sa force et sa faiblesse. Ce que le mythe nous dit, c'est qu'il est possible de s'inventer. II faut que l'humain se donne le monde à voir. Dès 
lors, il cesse d'être le spectateur passif des ombres sur les murs.

Platon dit : il n'y a pas que des ombres à contempler.

Peut-être que j'ai écrit cette chanson dans l'intention bien arrêtée de rattraper ceux et celles qui n'avaient rien vu de la beauté de cet écrit philosophique. Sortir la Caverne de la classe. Cette sorte de grotte pédagogique d'où il est parfois si difficile de faire entendre une voix venue de si loin. La classe, ce lieu clos d'où une parole nouvelle n'est pas audible dans le gémissement que font les cerveaux enchaînés.

Dans la Caverne, il y a toujours l'enfance. Ce monde peuplé de voix et de paysages. Cette boîte de carton que nous prenions pour une maison, une grotte ou la cache d'Ali-Baba. Ce couteau à pain qui servait à découper une toute petite fenêtre par laquelle on pouvait voir venir l'ennemi.

La Caverne est la plus belle légende de l'histoire de la philosophie. Celle qui explique le premier moment de la conscience. L'arrivée de la liberté. La venue du savoir.

Un enfant est poursuivi par des loups. II court. II sent les crocs proches de ses chevilles. II tombe. Les loups sont au-dessus de lui.

L'enfant se réveille. Il est dans sa chambre. Sa mère a laissé la porte entrebâillée. Une petite lumière pénètre dans la chambre. De la musique vient d'en bas. Le réel. Le vrai monde. Les vraies choses. La douceur du début de la nuit.

La Caverne, c'est tout autant le plaisir du dedans et le plaisir du dehors. 
La Caverne a mille formes. Elle est ce qui nous tient et ce qui nous délivre. Elle est le bonheur et le malheur. Elle est cette marche dans le difficile, cette promenade dans le doute, cette course folle vers la lumière. Le retour des ombres est toujours imminent.

On sort de la Caverne. Mais il y a un plaisir à y revenir. Les voyages qui ne sont pas racontés ne sont pas des voyages.

La Caverne, c'est l'invention et l'inventaire.

Je descendais lentement de mon lit. II ne fallait pas faire de bruit. Je sortais de ma chambre et j'allais m'asseoir juste en haut de l'escalier. Mon père était médecin, il terminait son bureau vers vingt-deux heures. Après, il s'installait dans le salon et écoutait de la musique. C'est du haut de cet escalier que j'ai rencontré Mouloudji, Brassens, Ferré, Montand, Greco, Bécaud, Patachou et bien d'autres...

La musique et la lecture pour se reposer du monde. Moi, la musique me tenait éveillé. La musique pour apaiser l'esprit. La musique pour mieux s'aimer. Mes parents ont toujours été des amoureux de la musique. L'amour se partage. Tout le monde dans la famille aime la musique.

J'écris en écoutant Marin Marais.

Le monde, avant de le voir, je l'ai d'abord entendu. Il est venu de la voix de ma mère et de mon père. L'écho du monde dans la caverne ou l'utérus. Le monde s'entend. Après il se voit.

La loi morale en nous et le Ciel étoilé au-dessus de nous! Kant!!!3

3. Ludwig van Beethoven, Camets intimes, traduction de M.V. Kubié, Paris :

Buchet Chastel, 1970, p. 6. 
Le philosophe invite à l'élévation. Le musicien invite à l'élévation. C'est ce mouvement de l'être qu'il faut viser. Sortir de toutes les cavernes en utilisant tout ce qui est possible pour retrouver ce ciel étoilé.

Vulgariser doit s'entendre noblement. II s'agit d'un voyage. II s'agit de mener ailleurs. On ne vulgarise pas pour piétiner. II s'agit d'ouvrir les yeux.

La loi morale doit être un guide. Le ciel étoilé est ce qu'il faut retrouver. Le ciel étoilé des idées. Le ciel étoilé du monde.

Philosopher, c'est naviguer entre les deux.

La musique est une main qui se pose sur le cœur. Elle le soulève ou elle l'étreint. Elle soigne ou blesse. Comme les mots, comme la vie. Tout est relié.

La musique est cette douceur ajoutée au monde. Une chanson peut nous réconcilier avec la vie, avec les autres. Elle n'est pas ce que l'on veut en faire aujourd'hui : une brisure entre les générations. Elle n'est pas ce qui sépare mais ce qui unit.

Elle peut être cette rencontre du jour et de la nuit.

La musique nous aide à penser, mais elle n'est jamais de la pensée.

II pleuvait. J'entendais très bien le bruit de la pluie sur le toit. Mais il y avait aussi un nocturne de Chopin. Des larmes s'ajoutèrent alors à la pluie sur le toit.

Chet Baker joue de la trompette comme un couple se souffle des mots dans l'oreille.

Qu'est-ce que la musique veut imiter puisque l'on dit que tout art est imitation? Une peine dans l'âme? Ce qui ne semblait pas pouvoir se partager? 
La musique s'accorde toujours au corps. Mais ce qui vient du corps passe par l'esprit, par l'âme.

Même une langue est un son. D'abord et surtout un son. Comme la musique. Elle permet de dire le monde. Le dehors et le dedans du monde.

Tout est son. Le vent. La mer. L'insecte.

La musique s'approprie les sons.

II y a toujours autre chose qu'un savoir dans la musique, dans une chanson. C'est la philosophie qui voudrait que tout le monde se réduise à un savoir.

"Je fais de la philosophie de contrebande», disait Michel Tournier dans un entretien avec le journaliste Stéphane Bureau. Faire une chanson sur la Caverne de Platon, c'est pour moi une sorte de contrebande philosophique. N'oublions pas ce que le mot vulgariser signifie : répandre (des connaissances) en mettant à la portée du grand public.

Ce n'est pas Platon qu'il fallait vulgariser, c'est l'Allégorie. Conserver l'essentiel. Le poétique et le philosophique. Rendre le philosophique chantant. Le refrain :

Mais d'où vient cette lumière

Cette chaleur sur ma peau

Quelque chose est là derrière

II me semble que c'est beau4

4. Refrain de la chanson La Caverne. Paroles de Marc Chabot, musique de Pierre Duchesne et Claire Pelletier, sur l'album de Claire Pelletier intitulé Murmures d'histoire, Disques Musi-Art, 1996. On trouvera en annexe, un extrait de l'Allégorie de la Caverne tiré de La République de Platon ainsi que l'intégrale du texte de la chanson La Caverne. 
Évidemment, chez Platon, il y a la raison. Mais le corps n'est pas là comme un concept qu'il faut repousser. Le philosophe aime les corps.

On ne passe pas sa vie à réclamer le Beau, toujours plus de Beau, si l'on est convaincu que le monde dans lequel nous vivons n'est que laideur.

Toujours chez Platon il y a la revendication du Beau.

Toujours chez Platon et pas assez chez les philosophes qui le lisent.

Ce qui serait vulgaire, ce serait d'imaginer Platon uniquement accessible aux universitaires.

Une chanson n'est qu'un petit voyage au pays du Beau. Celui ou celle qui le découvre comprend qu'on cherche à le faire entrer dans un monde. La chanson, c'est le seuil de l'infini. Léo Ferré mène à Baudelaire, Rimbaud, Apollinaire. La Caverne doit mener à Platon.

Demain ou dans dix ans, cela importe peu.

Les philosophes et les professeurs aiment s'occuper des choses mortes, des œuvres mortes. C'est un métier difficile, ingrat, celui de garder les choses et les œuvres vivantes.

Quand je dis à mes élèves que Nietzsche composait de la musique, qu'il y a même quelques compacts disponibles, c'est comme s'il se rapprochait d'eux. L'homme, le philosophe et l'œuvre. II devient plus humain.

Depuis que la musique rock est devenue une entreprise, une industrie culturelle, elle étouffe le cri de cette musique, la plainte du peuple. L'industrie culturelle fabrique des cris et des plaintes comme on fabrique des cure-dents. L'industrie culturelle arrache le cœur d'une musique, elle déforme le sens de nos 
cris. Elle ne conserve que le vulgaire.

Bien sûr que ça fait mal, mais ce n'est pas ce cri-là que nous voulons entendre.

La musique rassemble. N'importe qui, n'importe quand, n'importe comment, n'importe quoi, n'importe où.

L'exploitation de la vulgarité est trop souvent confondue avec la vulgarisation, l'accessible. Une mise à niveau des insignifiances, ce n'est pas de la vulgarisation, c'est une perte pour la musique, pour l'âme humaine, pour le plaisir, pour le bonheur.

Un rythme peut scander nos désirs, nos plaisirs, nos peines. Marche militaire, marche funèbre. Valse et mélancolie.

Toute musique est primitive.

Toute philosophie est un chant. Celui de la pensée. En philosophie règne l'ordre. II y a l'intention de faire sens à l'aide de la raison. En musique règne l'ordre, l'intention de faire sens à l'aide des sens.

Tout humain cherche à s'installer dans un langage. Le monde ne peut se dire autrement. Son univers intérieur ne peut exister sans cette mise en forme. Violence, douceur, frénésie, harmonie, bonheur, malheur et joie. Passion et raison.

Un homme s'assoit devant un piano. II pose ses mains sur le clavier. II ne maîtrise pas l'instrument, mais il joue, sachant fort bien que rien de ce qu'il ressent ne pourra se dire. II joue tout de même. Ce qu'il entend, ce n'est pas ce que nous entendons. Sa musique reste inaccessible. II joue et c'est n'importe quoi. Des sons bruts. Pourtant, en lui, une autre musique.

II y a toujours un humain qui s'assoit devant un piano et qui sait 
qu'il ne pourra pas se dire. Comment font-ils, les Chopin, Beethoven, Schubert ou Bach pour pleurer à l'aide d'un instrument?

Le poète, le philosophe s'assoient. Ils diront ce qu'ils sont avec cet instrument que l'on nomme le langage humain.

Je connais mes gammes. Mais je suis cet homme devant le piano. Je n'arrive pas à dire ce que je suis avec de la musique. Je caresse inutilement le clavier. Les mélodies sont en moi mais je n'ai pas les notes pour les faire entendre.

Toutes les violences incontrôlées sont le résultat d'un manque de vocabulaire. Le sang gicle. Les mots meurent.

Tous les blues se ressemblent. Un cri, une plainte. Le blues prend sa source dans la souffrance. Un Noir dans un champ de coton qui fabrique un chant et un son. Le blues ne s'écrivait pas, il n'y avait pas de crayons pour les esclaves.

J'ai vu une élève passer deux heures devant une feuille blanche. Parfois elle griffonnait quelques mots, finissait par se rebeller, jetait la feuille à la poubelle et retournait devant une autre feuille blanche.

Elle cherchait des mots. Elle voulait dire ce qu'elle pensait. Elle n'y arrivait pas. J'avais parfois le goût de lui crier : danse alors. Mais c'est un autre langage. Elle n'en possède aucun. Elle a presque vingt ans. Elle n'a aucun moyen pour se dire, pour se faire entendre. Elle sombre parfois dans la dépression.

"Tu n'auras rien", a-t-elle fini par me dire, les larmes aux yeux. Peut-être qu'elle écrira un jour parce qu'elle sait que, entre ce que nous avons dans la tête et ce qui vient au monde, il y a un autre monde. 
Je suis avec Richard Séguin à Saint-Venant-de-Paquet. Nous travaillons depuis deux jours. Nous nous levons très tôt le matin. Nous prenons un café. Nous parlons d'une chanson. II y a l'Amérique. II y a nos rêves. II y a le rêve américain. II a commencé une chanson, Journée d'Amérique. J'ai commencé une chanson, Dans nos villes d'Amérique. Les deux textes se rejoignent. Mais ni l'un ni l'autre ne sont une chanson. Nous le savons. Et nous tentons désespérément depuis deux jours de jumeler les mots. J'écris quelques lignes, il chante. Et nous retournons au silence. Ce n'est jamais ce que nous voulons.

En nous, colère et impatience. Nous en sommes à nos débuts. Nous nous connaissons peu. Nous cherchons des mots pour nous rejoindre. Nous voulons d'une chanson qui soit un nous.

Ce texte commun veut briser un silence. II y a plusieurs Amériques. Cette colère nous est commune. Elle est notre Amérique. Nous sommes deux, nous sommes dix, nous sommes mille.

C'est comme si tout ce monde voulait parler en même temps. Bousculade. Nous nous éparpillons dans la rage de dire.

Toute la journée à chercher quelques mots. La vie en Amérique n'est pas celle qu'on nous dit. Il y a des rêves qui n'aboutissent pas. II n'y a pas que le jet set.

The Lonesome Jubilee de John Cougar Mellencamp traîne près de l'appareil compact. Richard danse dans la cuisine. Je tape du pied. Nous rions.

Richard me dit : "On sort». II est tard. Rosie, sa chienne, se demande : c'est quoi l'idée d'aller se promener à cette heure? La nuit. Comme éclairage, les étoiles. II fait froid. Ça nous fait du bien de sortir. On retourne dans le monde. «Est-ce que tu imagines ce que c'est ce froid dans une ville d'Amérique? Loin de Vegas. Loin de l'argent. À quoi ça pense un homme qui est perdu dans la foule?"

Nous sommes en pleine campagne et nous ne sommes pas perdus. Nous sommes loin des humains et nous sommes avec les humains. 
Nous rentrons. Je lui demande de reprendre sa guitare. II chante Journée d'Amérique avec les mots que nous avons. Ce n'est pas ça. Je prends toutes les feuilles griffonnées durant la journée et je les jette. Richard crie. Moi aussi.

II nous faut un personnage. II nous faut un être de chair entre nous. C'est de lui que nous viendra la chanson. Un homme doit naître. II a des rêves. II y a le vent. La foule. Le chômage. Le travail au noir. La Loto, l'espoir.

Nous nous sommes couchés très tard en chantant Journée d'Amérique. "Demain matin si nous pouvons encore la chanter, ce sera une chanson".

Nous n'exprimons que les travers de l'univers.

Malgré tout, il nous faut trouver les bons mots. II y en a peu dans une chanson. Patience, exigence. Besoin d'une larme au coin de l'œil. Peut-être, mais il faut se méfier des larmes.

Une note qui ne vient pas au bout des doigts d'un musicien, est-ce aussi terrifiant qu'un mot qui ne vient pas au bout des doigts d'un écrivain?

Je travaille contre le cloisonnement des genres. Depuis longtemps. Peut-être depuis toujours. On ne peut pas passer sa vie à se demander : est-ce que je suis en train de faire de la philosophie? Le principal, c'est de faire. L'essentiel est là. Je n'écris pas pour épater les pairs. La philosophie n'a jamais réussi à tout dire. Mais elle est indispensable. Elle est une partie de notre héritage humain. C'est le même monde, mais avec du sens. La philosophie est une manière de danser sur le sens des mots. La philosophie rejoint la musique quand elle consent au plaisir.

Une chanson est un morceau de culture pour tout le monde. Elle ne va jamais vers tout le monde. Mais j'espère seulement qu'une chanson puisse faire grandir l'être. Dans la connaissance du monde, dans la connaissance de soi. 
Le rock hurle le désespoir de la jeunesse. Mais il est aussi ce qui exploite ce désespoir. Plus tard, on rencontre Brel, Brassens, Ferré. On se rend compte que le désespoir est là aussi. II est en permanence dans chaque être. II y a le vrai désespoir et celui qu'on nous vend. II faut choisir.

J'achève mes quarante ans. Parfois monte de moi une chanson de mon adolescence. Elle est là dans un coin de mon cerveau, parfaitement intacte. La complainte du marin de Clémence Desrochers. Claude Léveillée la chantait sur son tout premier disque.

Tu ne sauras jamais, jamais, chère Marie-Rose

Ce que j'ai enduré, tout seul, dans I'noir, su'l'pont

Pourtant c'était bien simpl', moi, j'voulais rien qu'une chose

J'voudrais tout simplement mêm'plus savoir ton nom 5

Tout le texte est en moi. Et je me revois presque immédiatement à Ayer's Cliff. Je marche près du lac Massawippi. Collégien du début des années soixante. Je retrouve une mélancolie depuis longtemps disparue. Aucune femme dans ma vie. Aucune histoire d'amour. La solitude.

Le texte philosophique, pour me plaire, doit être chantant. Nos idées doivent pouvoir danser. Philosopher, ce n'est pas s'acheter un classeur pour y ficher des concepts.

Ce serait trop simple.

La philosophie a besoin d'un rythme. C'est peut-être ce que Nietzsche avait compris.

5. Pour le texte complet de la chanson : Clémence Desrochers, Tout Clémence, tome $1: 1957-1974$, Montréal : VLB éditeur, 1993, p. 157. 
La pluie tambourine. Le vent siffle. Le tonnerre gronde. Les portes grincent, les chaises craquent et, dit-on, les murs ont des oreilles. Que serait l'humain sans musique?

La musique peut endormir, réveiller, hypnotiser, apaiser, aliéner, écraser, étourdir, caresser, conscientiser.

1945. Il y a eu le ballet de l'humiliation. La musique dans les camps. L'esthétique de la mort. La musique faisait son travail : endormir la douleur, étouffer les restes du cerveau, faire bouger des corps. Ordonner «l'anus du monde»6.

Nous avons quelques minutes seulement pour modifier l'âme des autres. Voilà le défi d'une chanson. C'est une opération pédagogique. Comme un cours de philosophie. Dans un cas comme dans l'autre, le résultat est vague. On entre dans une classe. II faut parler de Platon, de Thoreau, de Schopenhauer, de Nietzsche. II faut faire oublier que le monde dans lequel nous vivons existe. II faut que l'attention se porte ailleurs.

L'ange vagabond. Un texte hommage à l'écrivain Jack Kérouac. 1987. Quelque temps après un voyage à Lowell, Massachusetts. Sa ville natale. Je venais à peine de rencontrer Richard Séguin. II soupait à la maison avec sa compagne, Marthe Lévesque. Nous avions longuement parlé de Kérouac durant le souper. En soirée, nous avons écouté attentivement la bande sonore de l'émission Le sel de la semaine. Le seul entretien de Kérouac en français, avec Fernand Séguin.

Sylvain Lelièvre avait déjà écrit une chanson sur Kérouac. Une très belle chanson. II y avait aussi une autre chanson de CharlÉlie Couture : La route (oui mais Kérouac est mort). À l'époque, il y avait, à Québec, un Club Jack Kérouac. On y

6. "L'anus du monde" est l'expression qu'utilisaient les soldats nazis pour parler des camps. Sur le rôle de la musique dans les camps, voir Pascal Quignard, La haine de la musique, Paris : Calmann-Lévy, 1996. 
retrouvait quelques membres de sa famille. De Kamouraska et du bas du fleuve.

Nous voulions parler de notre appartenance à l'Amérique. Nous voulions dire l'univers culturel autrement.

Écrire sur la philosophie. Écrire sur une forme de discours. Se tenir juste à côté de ce discours. Se contenter de le montrer du doigt.

Écrire sur la musique ou la chanson. Écrire sur une forme de discours. Se tenir juste à côté. Se contenter d'affirmer : ouvrez un peu les oreilles.

Même l'écriture est un son.

Il y a des musiques qui font fuir la pensée.

Une musique ramène un souvenir troublant. Une chanson rappelle un fait. Elle est comme une photo, elle est comme un mot. Pouvons-nous faire autre chose que nous souvenir? Je ne sais pas.

Elle est enceinte. Elle écoute Barbara et sa tristesse. Elle se demande si l'enfant peut entendre. À l'intérieur d'elle vit un être. Elle chante Le mal de vivre. Elle chante. La nuit n'en finit plus. Elle pense à la vie. Le bonheur de l'être en elle. Le futur. La solitude. La sienne et celle de l'enfant.

Elle est enceinte et l'homme est parti lorsqu'il l'a su. Elle écoute Barbara. Elle cherche l'émerveillement. Une larme. Un rire. Elle cherche à comprendre. Elle ne sait rien de la vie et pourtant elle se prépare à la donner. Elle voudrait du bonheur immédiatement. Un tout petit bonheur comme celui qui consiste à chanter avec Barbara. Chanson triste, chanson gaie, cela n'a que très peu d'importance. Elle aimerait que la vie se chante. Elle 
aimerait que la vie en elle se danse.

Peut-on être autre chose qu'un esclave des sens?

La musique est le signe par excellence de la réminiscence.

Tolstoï : "Là où on veut avoir des esclaves, il faut le plus de musique possible". II y aurait mille réflexions à faire sur cette affirmation.

Peut-on dire qu'il y a en ce monde une angoisse du silence? La peur du vide. Cette peur combattue par la musique. Partout. Dans l'auto. Au travail. Partout. Un monde plein et vide à la fois.

II n'y a que la musique qu'on écoute qui existe. Toutes les autres dorment quelque part dans une discothèque. Nous les retrouverons, nous les redécouvrirons. Plus personne n'écoutait Marin Marais. Depuis le film Tous les matins du monde, Marin Marais a recommencé à exister. Nous ne pouvons pas faire contenir dans nos mémoires toute l'humanité, mais nous devons savoir que nous avons une mémoire. Sans elle, plus rien de nous.

Un petit air sur un piano, des mots sur du papier. Du travail. Du travail. Du travail. Et des mots qui restent dans nos tiroirs.

La chanson est l'un des derniers arts qui appartient à tout le monde, qui est sans école, qui n'a pas sa chaire dans nos universités, qui a la prétention de s'adresser à tout le monde, qui ne cible pas toujours son public, qui commence avec rien, qui se laisse avoir par les financiers, qui va directement au cœur, qui se moque du succès et qui ne pense qu'à ça.

Lire, écouter, penser, c'est le même geste. 
Philosophie et musique. Deux manières d'approcher la profondeur. Glisser hors du monde. Venir à l'autre.

\section{Marc Chabot \\ Beauport}

